\title{
THE INFLUENCE OF ROOT-END FILLING MATERIALS ON BONE HEALING - AN EXPERIMENTAL STUDY
}

\section{ANDREEA GULIE KUI ${ }^{1}$, ANTONELA BERAR ${ }^{1}$, LIANA LASCU ${ }^{1}$, POMPEI BOLFA ${ }^{2,3}$, BIANCA BOSCA ${ }^{4}$, CARMEN MIHU ${ }^{4}$, MIHAELA BACIUT ${ }^{5}$, RAMONA AVRAM ${ }^{6}$, MÂNDRA BADEA $^{6}$}

${ }^{1}$ Department of Prosthodontics, Faculty of Dentistry, Iuliu Hatieganu University of Medicine and Pharmacy, Cluj Napoca, Romania

${ }^{2}$ Pathology Department, University of Agricultural Sciences and Veterinary Medicine, Cluj Napoca, Romania

${ }^{3}$ Department of Biomedical Sciences, Ross University School of Veterinary Medicine, PO Box 334, Basseterre, St. Kitts and Nevis Federation, West Indies ${ }^{4}$ Department of Histology, Faculty of Medicine, Iuliu Hatieganu University of Medicine and Pharmacy, Cluj Napoca, Romania

${ }^{5}$ Department of Surgery and Implantology, Faculty of Dentistry, Iuliu Hatieganu University of Medicine and Pharmacy, Cluj Napoca, Romania ${ }^{6}$ Department of Dental Prevention, Faculty of Dentistry, Iuliu Hatieganu University of Medicine and Pharmacy, Cluj Napoca, Romania

\section{Abstract}

Aims. The aim of this experimental study is to assess the bone healing phenomenon produced in the presence of several dental materials: a polycarboxylate cement, a glass-ionomer cement, a composite resin and MTA (mineral trioxide aggregate) based cement.

Methods. The biocompatibility of four root-end fillings materials, used in periapical surgery was investigated after intra-osseous implantation of the materials in rats' calvaria. Tissue reaction was studied at 2, 4, 6, 8, 10 and 12 weeks after implantation. We took into consideration the presence of inflammatory cells (polymorphonuclear leukocytes, macrophages, plasma cells, lymphocytes and giant cells) and classified the aspects of the histological samples according to the following scale: 0 - no inflammation, 1 - mild, isolated inflammation, 2 - moderate, localized inflammatory reaction, 3 - severe, diffuse and intense inflammatory reaction.

Results. The inflammatory reaction was present at the six intervals for all the tested materials, but at 12 week interval, the reaction was minimal in all cases. Also, a dissolution reaction was observed for all the materials, less intense for glass-ionomer cement and polycarboxilate cement.

Conclusions. At the end of the experimental period, glass-ionomer cement and polycarboxilate cement suffered a lesser dissolution reaction as compared to the second group of tested materials.

Keywords: inflammation, root-end filling material, MTA, polycarboxilate cement, composite resin
Manuscript received: 02.10.2014

Accepted: 05.10.2014

Address for correspondence: gulie.andreea@umfcluj.ro

\section{Introduction}

One of the most used conservative surgical techniques in endodontics is apicoectomy. The studies collected over the years have succeeded in improving both surgical techniques, especially the dental materials used for 


\section{Dental Medicine}

root end filling and sealing of the apical surface $[1,2,3]$. Today, there are several types of materials used in endodontic surgery, either in orthograde filling or as a retrograde filling. All these materials must have certain characteristics like radioopacity, easy to be manipulated, non-absorbable, non toxic and well tolerated by periradicular tissues, among other qualities $[3,4]$. Numerous materials have been studied and used in apicoectomy, like zinc - oxide eugenol (ERM, IRM) cements, zinc phosphate cements, polycarboxylate cements, MTA (mineral trioxide aggregate) or composite resins [3].

In endodontic surgery the sealing materials that come in contact with the periapical tissues and the alveolar bone have an important role $[5,6,7,9]$. Over the years, numerous studies were published for various types of materials [10].

All materials used in endodontic surgery have an intimate contact with the surrounding host tissues, especially in case of root-end filling materials. For that reason it is very important to use a non-toxic material and biocompatible with the hard and soft tissues of the periodontium. There are several steps in evaluating the biocompatibility of dental materials, such as establishing the toxicity profile of these materials and evaluation of local toxicity. Among these tests, in vivo, subcutaneous, intra-osseous implantation tests have been developed for small laboratory animals. Despite of the large number of these tests, there are differences in tissues responses in different animal species and locations, making the results difficult to compare $[9,10]$.

The aim of this experimental study is to assess the bone healing phenomenon produced in the presence of several dental materials: a polycarboxylate cement, a glassionomer cement, a composite resin and MTA (mineral trioxide aggregate) based cement $[9,11,12,13,14]$.

\section{Materials and method}

In this study we have used as tested materials a polycarboxilate cement (PC) (Adhesor Carbofine SpofaDental $\left.{ }^{\circledR}\right)$, a glass-ionomer cement (GI) (Kavitan Plus - SpofaDental ${ }^{\circledR}$ ), a MTA based cement (MTA) (FillApex MTA - Angelus $\left.{ }^{\circledR}\right)$ and a dual-cured composite resin (CR) (Core-It SpiDent ${ }^{\circledR}$ ).

\section{Animals}

Adult male Wistar rats (weight between 180-200 grams) were used in this study. The study was approved by the Ethics Committee of the University of Medicine and Pharmacy "Iuliu Haţieganu", Cluj Napoca (Protocol No. 615/28.06.2012). All ethical principles of laboratory animals were followed. Animals were kept in a climatized room $\left(21-23^{\circ} \mathrm{C}\right)$ and received balanced food and water ad lib.

\section{Procedure}

In this study we used seventy rats. In preparation for the experimental procedure, the animals were anesthetized by an intramuscular injection containing Ketamine hydrochloride $(0.1 \mathrm{mg} / \mathrm{mL})$ in combination with Xylazine hydrochloride $(0.05 \mathrm{mg} / \mathrm{ml})$. After anaesthesia, a linear incision on the scalp was performed with \#15T, the skin was reflected and three osteotomies were performed (2.2 $\mathrm{mm}$ in diameter and $1 \mathrm{~mm}$ deep) using an inverted cone bur with $2 \mathrm{~mm}$ in diameter and with $1.0 \mathrm{~mm}$ in length cutting head under permanent cooling with saline solution $[7,8]$. The osteotomies were made in the same line, para-median, at $2 \mathrm{~mm}$ distance between them. The first orifice was left empty (control orifice), and the other two were filled with two of the four materials used in the study, so for the first group we implanted PC and GI and for the second group we used MTA and a dual-cured CR.

All the materials were prepared according to manufacturer specifications, we used sterile spatulas and plates for mixing the cements or we used the auto-mix syringe provided by the manufacturer of the materials. After irrigating with sterile saline solution for the first groups, the materials were delivered and compacted into the prepared osteotomy using dental condensers; as for the second group we delivered the materials using the auto-mix syringe for which the manufacturer provided them. During the surgery, while applying the materials in the orifices, we made sure not to create air bubbles or exceed the orifice with material. We sanitized the incision, we applied Oxytetracicline $12 \%$ powder over the incision and sutured with separate sutures, n 4.0 silk. The rats were monitored regularly for signs of infection and housed with food and water ad lib. Animals were euthanized using a lethal dose of Ketamine hydrochloride. Starting with week no. 2 after the surgery, 5 rats from each group were sacrificed every two weeks, meaning that in the $12^{\text {th }}$ week the last 5 rats from each group were euthanized. From each rat we harvested the calvaria which contained the three orifices. Bone samples were fixed in $10 \%$ neutral buffered formalin for 5 days. After this, the bone samples were made up to be introduced into small recipients. After that, the samples were washed in tap water for 40 minutes.

\section{Histology}

The next step was decalcifying the frontal bone samples, using a 1:1 solution of $8 \%$ Hydroclorhidric acid and $8 \%$ Formic acid. The samples were placed in a volume of at least 20 times greater than their own volume. Decalcifying solution was changed every two days until the process was completed. After the samples were decalcified they were washed with tap water for one hour. The bone samples were embedded in paraffin, sectioned at a thickness of $5 \mu \mathrm{m}$ and then stained with haematoxylin and eosin (H\&E). Histological preparations were examined and photographed using calibrated image retrieval by Olympus, and afterwards using an image acquisition and processing software Cell B (by Olympus).

Representative sections from each area to analyse were identified and photographed with the objectives $4 \mathrm{x}$, 10x, 20x and 40x. The images were evaluated histologically 
in terms of inflammation present around the area where the materials where implanted according to standard criteria $[7,8]$ The histological aspects of the orifices were included according to the adjacent inflammation near the implanted material (first in the area of contact with the pre-existing bone) or unfilled area (control orifice). We took into consideration the presence of inflammatory cells (polymorphonuclear leukocytes, macrophages, plasma cells, lymphocytes and giant cells) and classified the histological aspects of the samples according to the following scale: 0 - no inflammation, 1 - mild inflammation, isolated, 2 - moderate, localized inflammatory reaction, 3 severe, diffuse and intense inflammatory reaction.

\section{Results}

Sections from all specimens were obtained. For some samples the materials were removed while sectioning, therefore we chose for analysis 90 adequate samples for the first group and 90 adequate samples for the second group.

After quantifying the inflammatory lesions, the results obtained were analyzed for normality using the Shapiro-Wilk test. The data obtained were parametrically distributed and we used Student t-test to compare differences between control areas and areas with implanted biomaterials. We considered statistically significant values of $p<0.005$ to determine differences between the two filled orifices in each group and the control orifice. Statistical analysis was performed using the R software (The R Project for Statistical Computing).



Figure 1. In the first group, the control orifice and the orifices filled with materials are circled. The inflammatory reaction is absent or minimal for the two orifices filled with materials during the experimental period. Instead there is an inflammatory and fibrous reaction for the control orifice. Examination with a magnifying glass, $\mathrm{H} \& \mathrm{E}$.

\section{Control orifice MTA based cement Composite resin}

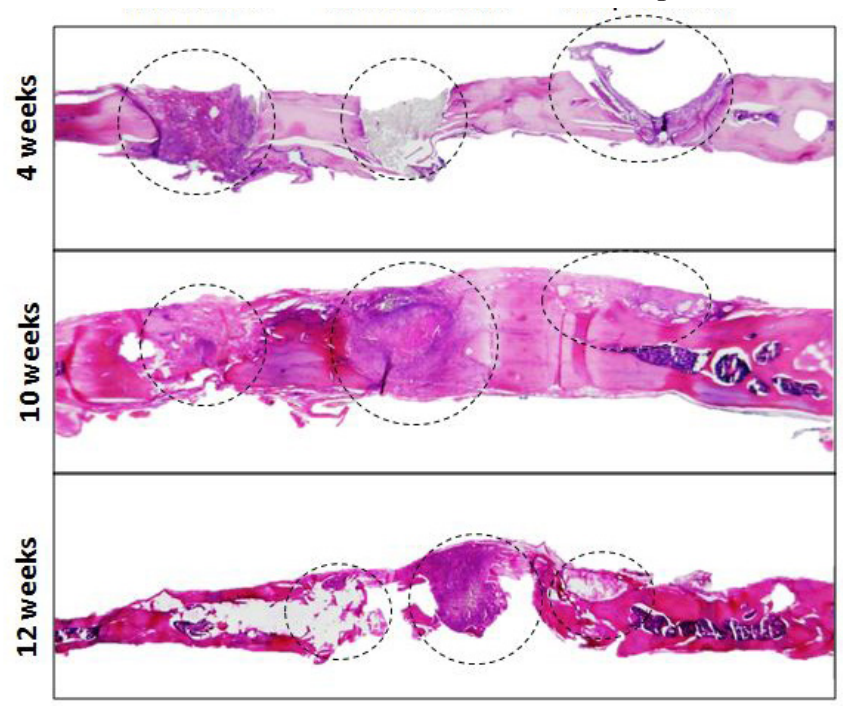

Figure 2. In the second group, the control orifice and the orifices filled with materials are circled. For the control orifice there is a progressive fibrous reaction, until the appearance of the bone at 12 weeks. In the case of MTA based cement after the 4 week interval an inflammatory response was not yet observed, while after 10 and 12 weeks an integration of the material in the reactive fibrous tissue was observed. Starting with the $2^{\text {nd }}$ week interval, for the composite resin orifice a fibrous reaction associated with the isolation of the material may be observed. Examination with a magnifying glass, $\mathrm{H} \& \mathrm{E}$.

\section{2 weeks}

For the control orifice, in both groups, a fibroinflammatory reaction was observed in all 10 animals euthanized, graded as mild or moderate. The inflammatory reaction for the control orifice was significantly important compared to all the areas of the four materials tested $(\mathrm{p}<0.01$; $\mathrm{p}=0.00767$ ). However, for all four tested biomaterials in most areas examined, there was no appearance of any inflammatory or fibrotic reactions.

\section{4 weeks}

At this interval a mild to moderate inflammatory and fibrotic reaction was observed for the orifices filled with the CR (composite resin) and a less intense reaction for PC (polycarboxilate cement), MTA and glass-ionomer cement. Inflammatory intensity differences were significant $(\mathrm{p}<0.05 ; \mathrm{p}=0.01692)$ for GI (glass-ionomer cement) and MTA compared to the control orifice (figure 1 and 2). Histologically, MTA had a finely granular aspect, grey colour and the GI had a hyaline texture.

\section{6 weeks}

Histological analysis of bone defects, with or without the test material showed the presence of a repair reaction tissue in all groups, but with different intensities. In both groups, the statistical differences were highly significant comparing all the four materials tested to control orifices in terms of inflammatory response. In the first group, we noticed a very high statistical difference between the 


\section{Dental Medicine}

control orifice, PC and GI ( $<<0,001 ; \mathrm{p}=0.000105)$. Also, in the second group (Fig. 4), the difference between the control orifice and MTA were highly significant $(\mathrm{p}<0.001$; $p=0.0004176$ ), but also between the control orifice and the resin composite cement $(\mathrm{p}<0.005 ; \mathrm{p}=0.001101)$. In case of MTA, at this point, the beginning of decomposition and phagocytosis of the material by inflammatory cells was observed.

\section{8 weeks}

At this interval, the inflammatory and fibrotic reaction decreased in intensity in all groups. It was observed that in most areas tested a fibrous tissue was infiltrating materials; the tested materials began their decomposition. (Fig. 3 and Fig. 4) We found significant differences in terms of inflammatory response between the areas with PC, MTA and the control orifice $(p<0.05 ; p=0.03183 ; p=0.04974)$.

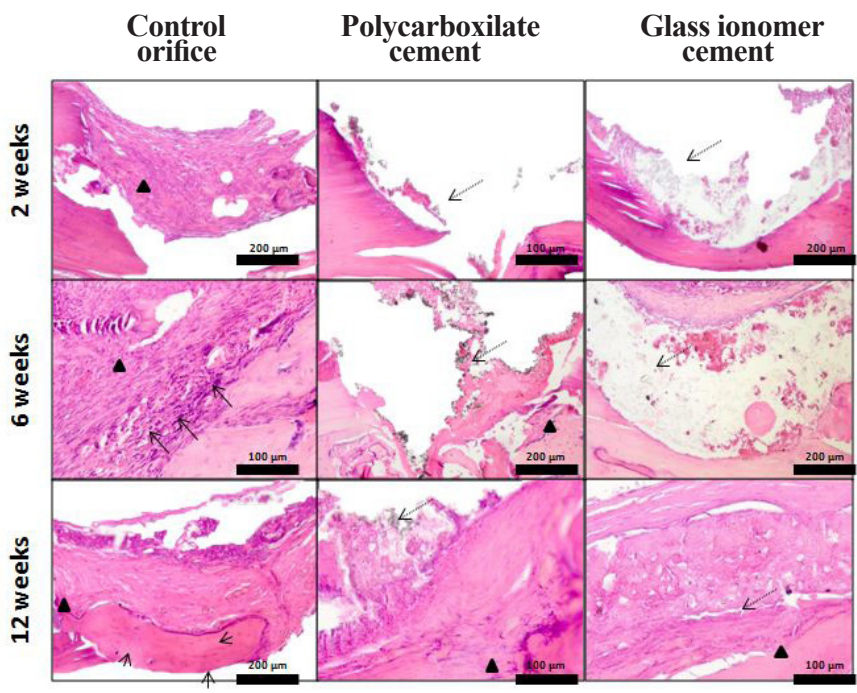

Figure 3. In the first group, for the control orifice a fibrous reaction was observed, which was supplemented after 6 weeks with the presence of mononuclear inflammatory cells (arrows) and osteoblasts, respectively; after 12 weeks, with the formation of a mature bone with osteocytes (arrowheads). Dotted arrows indicate the material tested. The fibrous reaction is marked with a black triangle. H \& E (40x).

\section{0 weeks}

The inflammatory reaction continued to decrease in intensity in all the areas studied. We found statistical differences only between the control orifice and PC and GI $(p<0.05 ; p=0.0203$ ). It was observed that for all samples analyzed the fibro-inflammatory reaction was minimal, fibrous tissue was better represented for the MTA and PC areas.

\section{2 weeks}

Although at this interval there were no statistical differences between the inflammatory response for the test areas and control orifice areas (the intensity was insignificant to absent), other changes were observed, however. For the control orifice areas, we observed new bone blades, while in the other areas it was much more discreet (for MTA, PC and GI cement) or not observed at all (CR).

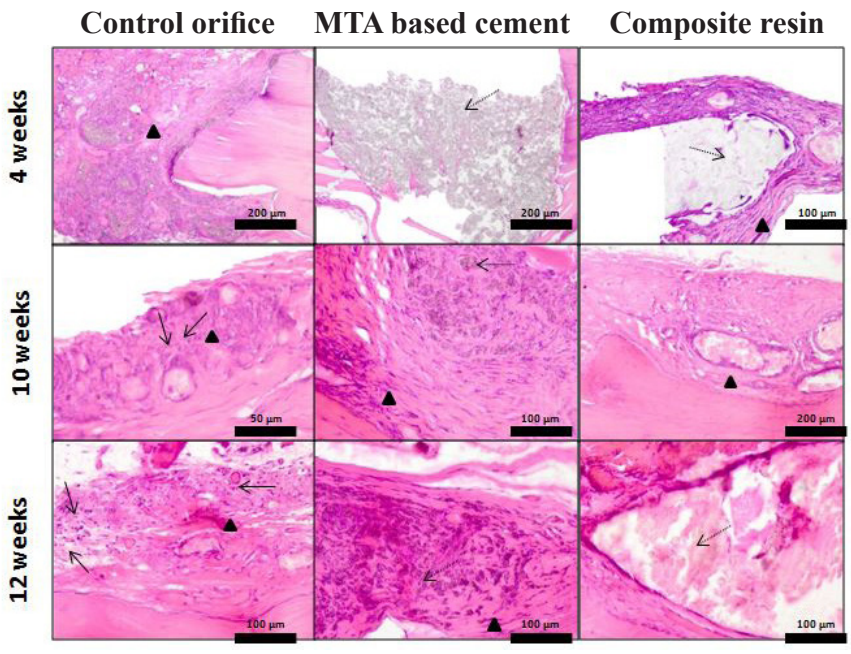

Figure 4. In the second group for the control orifice a fibrous reaction was observed, progressive and at the interval of 6 and 12 weeks inflammatory cells were also observed (arrows). Dotted arrows indicate the material tested. In this case, after 4 weeks, MTA based cement is seen clearly, but at the interval of 12 weeks it is in the process of decomposition by phagocytosis. Fibrous reaction is marked with a black triangle. $\mathrm{H} \& \mathrm{E}(40 \mathrm{x})$.

\section{Discussion}

Because well-documented as a model for evaluating bone healing, experimental studies on rat calvaria are good choices for testing the bone reaction in the presence of a root-end filling material used in apical surgery $[15,16]$.

For each group, the control orifice was one of the three orifices made in the calvaria, which was free of any material tested. Therefore, each sample had its own control area, which we considered significant in our study. We wanted to compare the natural process of bone healing with the areas filled with the materials tested at 6 different intervals $(2,4,6,8,10$ and 12 weeks).

After 2 weeks, for the control orifice a mild fibroinflammatory reaction was observed. Connective tissue proliferation was also observed departing from the periosteum. At the periphery of the defect a connective tissue with a weak cellularity and blood vessels of larger calibre developed, even in the presence of a material similar to osteoid. In the central area a young connective tissue was present, with good cellularity (fibroblasts, young collagen fibers and a few inflammatory cells, mainly macrophages) [17]. After 4 weeks, it was a reaction similar to that observed after 2 weeks, but with a higher fibrous reaction. At the periphery of the control orifice, a material similar to osteoid was present, and in the centre of the same orifice a necrotic material surrounded by a discrete inflammatory reaction was noted. After 6 weeks, the adult fibrous tissue from the periphery of the control orifice was observed advancing 
trough the centre where it a necrotic tissue surrounded by a moderate chronic inflammatory reaction (predominantly composed of mononuclear lymphocytes) was still present. The intensity of the fibro-inflammatory reaction was the highest observed at this interval, for the control orifice and for the other areas tested. Also, at the periphery of the control orifice area a multifocal, osteoid-like material was observed. At the interval of 10 weeks, similar to what we observed at 12 weeks, the fibrous reaction was more important, the central necrosis area decreased gradually in intensity, being replaced by fibrous tissue grown with a weak cellularity, rich in adult collagen and by new bone blades from the periphery to the central area [16].

For the PC (polycarboxilate cement) orifice no bone or fibrous reaction after 2 and 4 weeks was observed. After 6 weeks, there was a minimal fibrous reaction at the contact surface with the bone. At 8 weeks interval an inflammatory reaction around the test material, reduced in intensity, was present. A fibrous tissue was present at the periphery, but without bone reaction. Similar to the precedent situation, after 10 weeks, the test material began to be decomposed, surrounded by a fibrous reaction (adult fibrous tissue) and minimal inflammation (predominantly mononuclear lymphocytes). After 12 weeks, around the tested material, between the PC and the bone surface, a fibrous tissue was present, but in absence of any inflammatory reaction. In short, the inflammatory fibrous reaction around the tested material was microscopically visible at 6 weeks after the surgery and the minimum intensity of inflammation was observed at the end of the 12 week interval $[9,17]$. Regarding the decomposition of the material, the process was a slow one, from the periphery to the centre, and this process was visible after 8 weeks.

The second material analyzed in the first group was GI (glass-ionomer) cement, which had a hyaline aspect at the microscope. At the interval of 2 weeks no reaction was observed around the material. However, at 4 weeks, there was minimal fibrous reaction, focal, in the area between the material and the bone. The material began to decompose after 6 weeks without observing any fibro inflammatory reaction this time. In contrast, at the interval of 8 weeks, there was predominantly a fibrous reaction around the material with rare inflammatory cells and early decomposition of the test material. This decomposition process was also observed at the interval of 10 weeks, with a minimum intensity associated with a very discreet inflammatory reaction. After 12 weeks, the decomposition of the material continued, but the phenomenon was considered by comparison with the other tested materials, the fibrous reaction being more intense than in the previous period, also with a minimal inflammatory reaction. In brief, in the case of glass-ionomer cement, the intensity of the inflammatory response was minimal and constant during all six test intervals. The test material was surrounded by a fibrous reaction with minimum length observed initially until 8 weeks. GI started to be decomposed at 8 weeks, but the process was slow and gradual, from the periphery to the center $[9,17,19]$.

In the second group, the orifices filled with MTA did not show any inflammatory or fibrous bone response at the intervals of 2 and 4 weeks, the test material having a finely granular appearance, dark gray, which was sectioned easily. On the other hand, starting with the $6^{\text {th }}$ week it was observed that the inflammatory and fibrous reaction progressed until the interval of 12 weeks, a reaction that invaded the material, leading to a gradual decomposition of the MTA. The fibro-inflammatory reaction increased in intensity from 6 to 12 weeks, but it was minimal. The cells present at this level were the fibroblasts that infiltrated the material and the macrophages $[7,9,20]$. The reason for choosing an MTA was because of its setting times, of about 35 minutes, shorter than classical Grey or White MTA, an important aspect in case of an apicoectomy.

For the CR (composite resin) orifices, the aspect of the material was hyaline. At the interval of 2 weeks no reaction was observed around the tested material. Instead, at the 4 week interval a rather important fibro-inflammatory reaction was present, between the material and the bone. This consisted in a proliferation of a connective tissue in two areas (adult tissue with a weak cellularity with blood vessels of larger caliber on the outside and an area with young connective tissue, with a better cellularity) from the periphery to the center, with moderate congestion and the presence of an inflammatory infiltrate, predominantly represented by macrophages. Next, at 6,8 and at 10-weeks interval, the CR was gradually decomposed by the inflammatory and fibrous reaction, the material was microscopically observed as blocks of different sizes. In the area between the material and the bone, an adult fibrous tissue was observed starting with the 6 weeks-interval to 12 weeks, and the inflammatory reaction, although minimal, decreased in intensity gradually starting with the $8^{\text {th }}$ week until the $12^{\text {th }}$ week $[20,21]$.

There were two reasons establishing the testing period of 12 weeks. First, 4 weeks in a rat's life would be equivalent to approximately 120 weeks in human life (a life span of a rat is about 3 years and for humans is 90 years). The second reason is that the inflammatory reaction is minimal at the end of 12 week interval and it is unlikely that a subsequent inflammatory reaction would develop after this period [8].

\section{Conclusions}

None of the materials used in the study has been completely decomposed during the 12-week experiment. Decomposition reaction began quickly for PC (4 weeks) and for MTA (6 weeks) followed by GI and the CR ( 8 weeks). Bone repair reaction was most intense in the control area, up to 10 weeks, decreasing in intensity after the interval of 6 weeks being uniform in all groups at 12 
weeks interval. At the end of the experimental period glassionomer cement and polycarboxylate cement were less decomposed as compared to the second group of tested materials.

\section{Clinical relevance}

For the four tested materials a biocompatibility level based on the histological findings and the biocompatibility scale that has also been described in other studies may be assigned. Although all the four materials are considered biocompatible, the glass-ionomer cement and polycarboxilate cement were less decomposed than the other two materials; this fact has a clinical relevance, when the root-end cavity must be sealed for as long as possible and dissolution of the filling material is not desired. We acknowledge the limits of this study, therefore other experiments must be conducted in the future.

\section{References}

1. Regan JD, Gutmann JL, Witherspoon DE. Comparison of Diaket and MTA when used as root-end filling materials to support regeneration of the periradicular tissues. Int Endod J. 2002;35(10):840-847.

2. Berne C. Chirurgie périradiculaire. Rev Mens Suisse Odontostomatol. 2001;111:586-590.

3. Wilhelm-Joseph Pertot, Horace Siméoni. Chirurgie périapicale. EMC Consult. 1994. 1-9.

4. Lindeboom JAH, Frenken JWFH, Kroon FHM. A comparative prospective randomized clinical study of MTA and IRM as rootend filling materials in single-rooted teeth in endodontic surgery. Oral Surg Oral Med Pathol Oral Radiol Endod. 2005;100:495500 .

5. Torabinejad M, Parirokh M. Mineral trioxide aggregate: a comprehensive literature review - Part II: Leakage and biocompatibility investigations. J Endod. 2010;36:190-202.

6. Cunha SA, Rached FJA, Alfredo E, León JE, Perez DE. Biocompatibility of sealers used in apical surgery: a histological study in rat subcutaneous tissue. Braz Dent J. 2011;20(1):299305.

7. McNamara RP, Henry MA, Schindler WG, Hargreaves KM. Biocompatibility of accelerated mineral trioxide aggregate in a rat model. J. Endod. 2010;36(11):1851-1855.

8. Moretton TR, Brown CE, Legan JJ, Kafrawy AH. Tissue reactions after subcutaneous and intraosseous implantation of mineral trioxide aggregate and ethoxybenzoic acid cement. J Biomed Mater Res. 2000;52(3):528-533.

9. Torabinejad M, Pitt Ford TR. Root end filling materials: a review. Endod Dent Traumatol 1996;12(4):161-178.

10. Tanomaru-Filho M, Luis MR, Leonardo MR, Tanomaru JMG, Silva LAB. Evaluation of periapical repair following retrograde filling with different root-end filling materials in dog teeth with periapical lesions. Oral Surg Oral Med Oral Pathol Oral Radiol Endod. 2006;102:127-132.

11. Gomes-Filho JE, Watanabe S, Lodi CS, Cintra LT, Nery MJ, Filho JA, et al. Rat tissue reaction to MTA FILLAPEX ${ }^{\circledR}$. Dent Traumatol. 2011;28(6):452-456.

12. Puertas F, Santos H, Palacios M, Martı S. Polycarboxylate superplasticiser admixtures: effect on hydration, microstructure and rheological behaviour in cement pastes. Adv Cem Res. 2005;17(2):77-89.

13. Al-Badri IA, Kamel FM. Clinical use of glass-ionomer cement: A Literature Review. The Saud Dent J. 1994;6:107-116. 14. Hervás-García A, Martínez-Lozano MA, Cabanes-Vila J, Barjau-Escribano A, Fos-Galve P. Composite resins. A review of the materials and clinical indications. Med Oral Patol Oral Cir Bucal. 2006;11:E215-220.

15. Porto GG, Vasconcelos BC, Andrade ES, Carneiro SC, Frota MS. Is a $5 \mathrm{~mm}$ rat calvarium defect really critical? Acta Cir Bras. 2012;27(11):757-760.

16. Develioglu H, Unver Saraydin S, Kartal U. The bone-healing effect of a xenograft in a rat calvarial defect model. Dent Mater J. 2009;28:396-400.

17. Priyanka SR. A Literature Review of Root-End Filling Materials. IOSR Journal of Dental and Medical Sciences. 2013;9(4):20-25.

18. Carvalho LRP, Breyner NM, Hell RCR, Valério P, Novikoff S, Goes AM. Healing Pattern in Calvarial Bone Defects Following Bone Regeneration in Rats Guided by Chitosan Scaffold and Adipose Tissue-Derived Mesenchymal Stem Cells. Open Tissue Eng Regen Med J. 2012;5:25-34.

19. Niederman R, Theodosopoulou JN. A systematic review of in vivo retrograde obturation materials. Int Endod J. 2003;36:577586.

20. Otani K, Sugaya T, Tomita M, et al. Healing of experimental apical periodontitis after apicoectomy using different sealing materials on the resected root end. Dent Mater J. 201130:485492.

21. Ozbas H, Yaltirik M, Bilgic B, Issever H. Reactions of connective tissue to compomers, composite and amalgam rootend filling materials. Int Endod J. 2003;36:281-288. 